https://helda.helsinki.fi

Kaposi's sarcoma herpesvirus-induced endothelial cell reprogramming supports viral persistence and contributes to Kaposi's sarcoma tumorigenesis

\title{
Gramolelli, Silvia
}

2017-10

Gramolelli , S \& Ojala , P M 2017 , ' Kaposi's sarcoma herpesvirus-induced endothelial cell reprogramming supports viral persistence and contributes to Kaposi's sarcoma tumorigenesis ' , Current opinion in virology , vol. 26 , pp. 156-162 . https://doi.org/10.1016/j.coviro.2017.09.002

http://hdl.handle.net/10138/232265

https://doi.org/10.1016/j.coviro.2017.09.002

publishedVersion

Downloaded from Helda, University of Helsinki institutional repository.

This is an electronic reprint of the original article.

This reprint may differ from the original in pagination and typographic detail.

Please cite the original version. 


\section{Kaposi's sarcoma herpesvirus-induced endothelial cell reprogramming supports viral persistence and contributes to Kaposi's sarcoma tumorigenesis Silvia Gramolelli ${ }^{1}$ and Päivi M Ojala ${ }^{1,2,3}$}

Kaposi's sarcoma (KS) is an endothelial tumor causally linked to Kaposi's sarcoma herpesvirus (KSHV) infection. At early stages of KS, inflammation and aberrant neoangiogenesis are predominant, while at late stages the disease is characterized by the proliferation of KSHVinfected spindle cells (SC). Since KSHV infection modifies the endothelial cell (EC) identity, the origin of SCs remains elusive. Yet, pieces of evidence indicate the lymphatic origin. KSHV-infected ECs display increased proliferative, angiogenic and migratory capacities which account for KS oncogenesis. Here we propose a model in which KSHV reprograms the EC identity, induces DNA damage and establishes a dysregulated gene expression program involving interplay of latent and lytic genes allowing continuous reinfection of ECs attracted to the tumor by the secretion of virus-induced cellular factors.

\footnotetext{
Addresses

${ }^{1}$ Research Programs Unit, Translational Cancer Biology, University of Helsinki, Biomedicum Helsinki, P.O. Box 63 (Haartmaninkatu 8), University of Helsinki FIN-00014, Finland

${ }^{2}$ Foundation for the Finnish Cancer Institute, Helsinki, Finland

${ }^{3}$ Section of Virology, Division of Infectious Diseases, Department of Medicine, Imperial College London, London, UK

Corresponding author: Ojala, Päivi M (paivi.ojala@helsinki.fi)
}

Current Opinion in Virology 2017, 26:156-162

This review comes from a themed issue on Viruses and Cancer

Edited by Ethel Cesarman and Thomas Schulz

For a complete overview see the Issue and the Editorial

Available online 12th October 2017

http://dx.doi.org/10.1016/j.coviro.2017.09.002

1879-6257/@ 2017 Elsevier B.V. All rights reserved.

\section{Kaposi's sarcoma and identification of its associated herpesvirus}

Kaposi's sarcoma (KS) was named after a Hungarian dermatologist, Moritz Kaposi, who first described five cases of this multifocal, pigmented tumor of the skin. KS remained a rare disease until the 1980 s when an aggressive form was observed among the AIDS patients. The high number of cases together with the bad prognosis associated with AIDS-KS prompted researchers on studying the disease in detail. Particular efforts were put into the identification of KS causal agent and in 1994 Chang and Moore identified it as a newly discovered virus named Kaposi's sarcoma herpesvirus (KSHV) [1]. Classified as a gamma2 herpesvirus [2], KSHV has been subsequently associated also with two lymphoproliferative disorders: primary effusion lymphoma (PEL) and multicentric castleman disease (MCD) $[3,4]$.

KSHV displays a biphasic life cycle with a latent and a lytic phase. Although latency represents the default mode of persistence in the tumor cells, both phases contribute to KS pathogenesis. Since many reviews [5-8] recently discussed the molecular mechanisms of $\mathrm{KS}$ induced tumorigenesis, here we will focus on the reprogramming and manipulation of endothelial cells and its contribution to KS pathogenesis.

\section{KS: an angiogenic, inflammatory tumor of the endothelium}

Different forms of KS can be distinguished according to their epidemiological characteristics. In the sub-Saharan Africa KS is a common tumor among men and children (epidemic KS), whereas in the Mediterranean basin a milder form of KS affects primarily elderly men (endemic KS) [9]. The clinical features of KS become more aggressive when it occurs in immunosuppressed patients, such as transplant recipients (iatrogenic KS) and AIDS patients (AIDS KS) [10,11]. In these individuals, the lesions are life threatening because of the visceral involvement [11].

Microscopically, the different forms of KS are indistinguishable and display rather stage-specific features. At the early phase, KS manifests as patches characterized by abundant and aberrant angiogenesis. The abnormal blood vessels are leaky, thus accounting for the extravasation of erythrocytes and inflammatory cells that populate the lesion (Figure 1). The early KS patch appears as a flat, red macula (located in the limbs or face) and no evident tumor mass is present. In these patches, as little as $10 \%$ of the tumor is composed of KSHV-infected cells. When progressing toward advanced stages, KS develops into plaques and then into nodules. Here, $90 \%$ of the cells are KSHV-positive. The tumor mass is macroscopically evident and composed of so-called spindle cells (SCs) [12] that is, KSHV-infected cells 


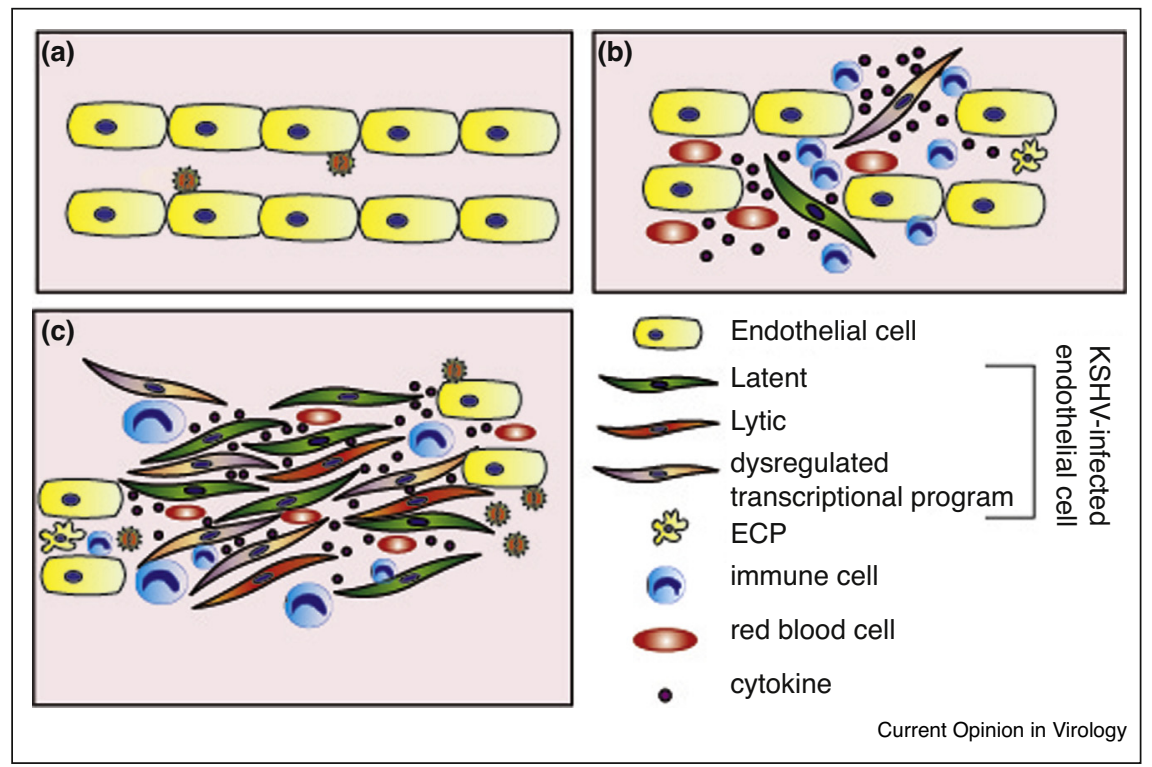

Schematic model of a KS lesion (a) KSHV virions infect endothelial cells. (b) Early KS lesion: KSHV infected endothelial cells display the typical spindle morphology and produce pro-angiogenic and inflammatory cytokines that chemotactically attract immune cells to the tumor site. Aberrant angiogenesis takes also place and causes the extravasation and accumulation of red blood cells. (c) Late KS lesion: the tumor is composed of KSHV-infected SCs. Majority of the SC are in a latent/dysregulated lytic phase, produce inflammatory and angiogenic chemokines and express many oncogenic viral proteins. Only a small subset of SCs are in a lytic phase and they produce new infectious virions for the maintenance of the population of infected cells.

with characteristic, elongated (spindle) morphology (Figure 1).

\section{On the cellular origin of $\mathrm{KS}$ spindle cells}

Different studies reported that SCs express a number of endothelial cell (EC) markers, for example, CD31, CD34 and factor VIII but they also harbor, to a lesser extent, lineage specific signatures of smooth muscle cells ( $\alpha$-SMA), monocytes/macrophages, dendritic cells as well as fibroblasts and mesenchymal cells $\left[13,14^{\bullet \bullet}, 15-18\right]$.

The gene expression signature of KS SCs indicates an endothelial origin; however, it is still a matter of debate whether the original precursor belongs to the blood or lymphatic lineage. KSHV infection of lymphatic ECs (LECs) triggers the reprogramming toward a less differentiated, immature blood ECs (BECs) phenotype, making KSHV-LECs more similar to BECs than their uninfected counterpart. KSHV-infected BECs, vice versa, express lower levels of blood vascular markers such as CXCR4 and Neuropilin-1 and show an increase in lymphatic markers like Prox1, VEGFR3, podoplanin and LYVE1 (reviewed in [19]). KSHV infection of ECs manipulates the EC differentiation program driving each cell type away from its original, mature phenotype and pushing it toward an undifferentiated, even mesenchymal-like cell type with mixed identity (reviewed in $\left[20^{\circ}\right]$ ). As a result,
KSHV-infected ECs display tumorigenic properties such as increased angiogenic, invasive and migratory abilities and, although not fully immortalized [21,22], they exhibit a growth advantage over their uninfected counterparts $\left[14^{\bullet \bullet}, 23\right]$.

Several observations suggest that LECs rather than BECs are the main precursor of SCs. First, the localization of KS lesions in tissues rich in lymphatics (e.g. skin and mucosa) [24-26]. Second, KSHV-LECs exhibit, in culture, the elongated (spindling) morphology, resembling KS-SCs, whereas in BECs the morphology is not significantly altered by KSHV infection. Third, LECs can be more efficiently infected and harbor a higher viral copy number [13]. In Addition, the comparison of the gene expression profile of nodular KS lesion with those of BECs and LECs showed that KS signature resembles, at least at this stage, more LECs than BECs [13]. Moreover, three-dimensional culture of KSHV-LEC as spheroids has been shown to recapitulate many of the features found in the KS lesions, among others the differential expression of endothelial and mesenchymal markers in virusinfected cells and the increased tumorigenic invasiveness in the $3 \mathrm{D}$ matrix $\left[14^{\bullet \bullet}\right]$.

Still, the presence of KSHV-infected BECs in KS lesions cannot be ruled out. KSHV-BECs are found in the early 
KS patches, where virus-positive cells are lining the walls of aberrant blood vessels. However, in the advanced lesions, although representing the majority of the tumor mass, KSHV-positive SCs were not found in the blood vessels [27]. One could hypothesize that KSHV infects both BEC and LEC at the early stages of the disease, but then KSHV-LECs, more susceptible hosts for the virus, propagate more efficiently and become the predominant cell type in advanced lesions. In agreement with this hypothesis is a study in which Prox1 expression, a marker of lymphatic differentiation (see below), was investigated in AIDS-oral KS. Here, Prox1, almost absent in the early KS patches, increased significantly in the advanced plaque and nodular stages [28 $8^{\circ}$.

Another possibility is that KS-SCs arise from the infection of circulating EC precursors (ECPs), CD34-positive bone marrow-derived cells that home into angiogenic sites where they differentiate into mature ECs [19]. In support of this hypothesis: first, ECPs isolated from KS patients were found positive for KSHV, retained the virus after several passages and were able to sustain lytic replication [29 $9^{\bullet \bullet}$; second, ECPs from KS patients displayed higher angiogenic potential in vitro [30 ${ }^{\circ}$; third, KSHV-positive, CD34+, adherent SCs were found in the blood mononuclear cell fraction [31]; fourth, the multifocal nature of KS lesions occurring independently in different body areas and preferentially in the surgical scares [25,32] or other sites of previous inflammation and angiogenesis, where the ECPs are recruited.

\section{Molecular mechanisms of KSHV-induced trans-differentiation of endothelial cells}

The trans-differentiation induced by KSHV represents an abnormal pathological process that can endow ECs with tumorigenic properties (increased proliferation, invasiveness and angiogenic potential), thus contributing to $\mathrm{KS}$ oncogenesis. In order to manipulate the EC-lineage identity, KSHV infection interferes with the expression of factors responsible for the differentiation and maintenance of specific EC types.

The transcription factor prospero homeobox 1 (Prox1) has been identified as the master regulator of the LEC fate [33]. Prox1 is required for the development of the lymphatic vasculature [34,35], and it also maintains the LEC cellular identity $[36,37]$. Ectopic expression of Prox1 in BECs drives the expression of LEC-specific markers (podoplanin, VEGFR3, LYVE1) and suppresses those accountable for the BEC signature [33,38].

KSHV modulates Prox1 expression both in LECs and BECs with opposing results $\left[13,14^{\bullet \bullet}, 19,39,40\right]$. In BECs Prox1 is not expressed under normal conditions, however, upon KSHV infection, Prox1 expression is induced and activates at least part of the LEC-differentiation program. In LECs, normally expressing high levels of Prox1, KSHV infection results in the downregulation of this transcription factor. The molecular mechanisms that govern Prox1 levels during KSHV infection have been recently elucidated. In KSHV-BECs Prox1 expression is driven by the virus-induced upregulation of IL3R $\alpha$, which controls Prox1 through Stat5. In KSHV-LECs, however, IL3R $\alpha$ does not affect the already high Prox1 expression that is regulated by the Notch1-Hey1 circuit. The KSHV-driven activation of Notch1, triggered by the viral proteins vFLIP and vGPCR $\left[14^{\bullet \bullet}, 41\right]$, is responsible for the downregulation of Prox1 in infected LECs but does not affect Prox1 levels in KSHV-BECs. Thus, the simultaneous activation of Notch1 and ILR3 $\alpha$ signaling by KSHV, which contributes to the differential regulation of Prox1 levels, leads to lymphatic reprogramming of BECs and induces blood vascular differentiation in LECs.

Another transcription factor with a pivotal role in KSHVinduced trans-differentiation of ECs is the musculo-aponeurotic-fibrosarcoma oncogene homolog (MAF). Expressed in LECs but not in BECs, MAF represses the BEC-specific genes, thus contributing to the maintenance of the LEG identity $[38,39]$. In a study aiming to identify new KSHV miRNA targets in LECs, MAF was among the top three downregulated genes upon ectopic expression of the KSHV miRNA cluster [42]. Further analysis showed that suppression of MAF in KSHV-LECs triggered the upregulation of BEC-specific genes such as GXCR4, FTL1 and GXCL12. Although no direct interaction has been shown between MAF and Prox1 it has been speculated that these transcription factors might form a repressor complex that contributes to the suppression of the BEC markers to maintain the LEC phenotype.

\section{Inflammatory microenvironment, lytic reactivation and the unique, viral transcriptional program in LECs}

KSHV lytic reactivation is crucial for the virus-induced tumorigenesis. This is demonstrated by the high viral loads in plasma associated with the worst KS prognosis and the clinical data indicating that inhibitors of lytic replication (i.e. ganciclovir and forscarnet) have a preventive effect on KS recurrence [43-45]. However, given that reactivation occurs in as little as $2 \%$ of the SCs and that upon completion of productive lytic replication phase KSHV-infected cell dies [46], how can an event occurring in such a small and destined to die cell population be important for tumorigenesis? To explain these apparently contradictory facts, a so-called paracrine effect has been hypothesized [47]. During the lytic phase virtually all viral ORFs are expressed. Several ORFs can manipulate the cellular signaling thus inducing the secretion of proinflammatory and angiogenic factors and modulating the behavior of SCs in the tumor microenvironment. For instance, ectopic expression of a lytic KSHV protein vGPCR in ECs in vitro and in vivo increases the expression and secretion of several angiogenic and inflammatory 
factor [48]. However, KSHV is known to elicit a systematic shut-off of cellular transcripts during the lytic phase $\left[49^{\bullet \bullet}\right]$. Therefore, the viral manipulation of the host secretome upon expression of a single viral protein may not faithfully recapitulate what happens in virusinfected cells during lytic replication. Although some transcripts for cytokines (e.g. IL6, IL1) can escape the shut-off, a dramatic reduction in the induction of proinflammatory and angiogenic factors was observed when comparing lytic cells expressing vGPCR versus KSHVinfected cells $\left[50^{\circ}, 51\right]$.

Lytic reactivation is also important to maintain a population of infected cells within the tumor. ECs, in fact, inefficiently maintain the latent infection and they lose the episome after a few rounds of cell division both in vivo and in vitro $\left[52^{\circ \bullet}\right]$. Lytic replication therefore would be needed to constantly produce new virions that can infect the uninfected cells in the affected tissue. This can be seen as an interesting strategy evolved by the virus to survive and successfully establish a life-long latent infection in a cell type (endothelial) that the virus is unable to immortalize. Despite numerous attempts to propagate cultures of KS biopsies, there are no patient-derived endothelial cell lines stably harboring KSHV [53-56], while several B cell lines stably and latently infected with KSHV have been established from PEL patients [57]. These experimental observations support the notion that, in order to survive in certain cell types, KSHV adopts strategies that are different from those developed for infected B cells.

Several reports have suggested that, in some cellular contexts a dysregulated viral transcriptional program exists that is not ascertainable to either of the latent or lytic viral phases $\left[58,59,60^{\bullet}, 61\right]$. This dysregulated gene expression program is characterized by sporadic expression of lytic markers without efficient production of virions and it was initially observed upon KSHV infection of murine cells [58,59]. In particular, murine bone marrow-derived endothelial progenitors expressed many lytic markers upon KSHV infection, and could generate KSlike tumors in mice [58]. However, the relevance of these models has been questioned since mice are not permissive hosts for KSHV.

More recently, a similar viral transcriptional program has been described also in KSHV-LECs [60 $\left.{ }^{\circ}\right]$. Upon infection, differently from BECs, where only the latent genes were detected, LECs displayed a dysregulated viral gene expression pattern. Surprisingly, the KSHV-LECs expressed a variety of lytic ORFs and continued the host cell proliferation, normally not observed during productive lytic replication cycle. This 'leakage' of lytic transcripts in LECs suggests that this could represent an abortive lytic program not producing new virions and not leading to cell death. Although not specifically addressed in the study, it is tempting to speculate that virus-induced host shut-off is not fully effective in the KSHV-LECs, thus allowing the ongoing cell proliferation and perhaps secretion of inflammatory and angiogenic factors. Furthermore, a small amount of infectious progeny was produced, suggesting that a minority of KSHVLECs spontaneously underwent full lytic replication cycle. Despite the low viral titers, the pool of spontaneously produced progeny virions might be sufficient to expand the population of infected cells and to compensate for the loss of KSHV episomes in dividing cells.

\section{Contribution of the DNA damage response (DDR) to KSHV pathobiology}

The association between KSHV infection and KS, PEL and MCD undoubtedly ascribes KSHV to the oncogenic viruses. However, KSHV infection does not induce cellular immortalization, thus suggesting that virus persistence is required to initiate and support tumorigenesis. Activation and manipulation of DDR, a known hallmark of cancers in general [62] represents also a common theme in viral oncogenesis $[63,64]$. DDR is activated also in the spindle cells in KS lesions, especially in the early (patch) and, to a lesser extent, late (nodular) stages [65\%]. This observed checkpoint activation can at least partially explain the low proliferative index of the spindle cells in the KS tumors.

In vitro, DDR has been shown to be induced both during latency establishment and lytic replication phase. During primary infection of ECs, DDR is mounted as early as 30 min post infection. Interestingly, the DDR marker gamma H2AX is localized to the viral genome right after its delivery to the nucleus. This KSHV-induced DDR appeared to be essential for latent gene expression and episome replication [66]. In addition, we and others have reported that in different KSHV-infection models the DDR is instigated upon lytic reactivation [67-69], thus suggesting that the virus replication induces a genomic stress that might represent a driving force in tumorigenesis or that DDR could provide a favorable host cell environment for efficient virus replication.

Although it is known that DDR, first, can function both as an anti-cancer barrier oras a driving force in tumorigenesis, second, is elicited within KS lesions and third, is activated during KSHV lytic replication, the extent of its contribution to KSHV tumorigenesis remains to be elucidated.

\section{Future perspectives}

Evidence coming from epidemiological, clinical and in vitro studies supports the lymphatic origin of SCs. Here we suggest that KSHV reprograms EC identity to establish a viral gene expression program that allows production of new virions for continuous reinfection of ECs (or ECPs) to maintain a persistent infection (Figure 1). In 
addition, the DDR elicited and usurped by the virus to promote its own persistence (in latency) and replication (in the lytic phase), contributes to KS tumorigenesis.

If occurring within a KS lesion, such a model might explain several features of the disease: first, the occurrence of sporadic lytic markers in KS biopsies (due to the LEC-specific viral gene expression program and/or spontaneous lytic reactivation); second, the presence of an angiogenic and inflammatory microenviroment (induced by the sporadic expression of lytic viral genes); third, the maintenance of a population of KSHV-infected cells within the lesion (due to the low-rate spontaneous production of virions); fourth, the presence of DDR activation and low proliferative index in KS lesions. A recent reportsuggests that such a dysregulated transcriptional program occurs at least in a subset of AIDS-KS patients $\left[70^{\circ \bullet}\right]$. Viral profiling of KS biopsies showed that in some tumors a tight latent program was present, whereas in a fraction of the biopsies the viral transcriptional program included expression of both latent and lytic ORFs.

However, several issues remain unclear. For instance, what is the role of circulating KSHV-positive ECPs, how to conceive the 'leaky' lytic program with the virus host shut-off, and what is the role of KSHV reprogramming in the context of virus persistence and what is the contribution of DDR activation and the consequent genomic stress to KS oncogenesis?

\section{Funding}

This work was supported by the Academy of Finland, Sigrid Juselius Foundation, Finnish Cancer Foundation and K. Albin Johansson Foundation.

\section{Conflict of interest}

The authors declare no conflict of interest.

\section{References and recommended reading}

Papers of particular interest, published within the period of review, have been highlighted as:

- of special interest

$\bullet$ of outstanding interest

1. Chang Y, Cesarman E, Pessin MS, Lee F, Culpepper J, Knowles DM, Moore PS: Identification of herpesvirus-like DNA sequences in AIDS-associated Kaposi's sarcoma. Science 1994, 266:1865-1869.

2. Russo JJ, Bohenzky RA, Chien MC, Chen J, Yan M, Maddalena D, Parry JP, Peruzzi D, Edelman IS, Chang Y et al.: Nucleotide sequence of the Kaposi sarcoma-associated herpesvirus (HHV8). Proc Natl Acad Sci U S A 1996, 93:14862-14867.

3. Cesarman E, Chang Y, Moore PS, Said JW, Knowles DM: Kaposi's sarcoma-associated herpesvirus-like DNA sequences in AIDS-related body-cavity-based lymphomas. N Engl J Med 1995, 332:1186-1191.

4. Soulier J, Grollet L, Oksenhendler E, Cacoub P, Cazals-Hatem D, Babinet P, d'Agay MF, Clauvel JP, Raphael M, Degos L et al.: Kaposi's sarcoma-associated herpesvirus-like DNA sequences in multicentric Castleman's disease. Blood 1995, 86:1276-1280.
5. Wong JP, Damania B: Modulation of oncogenic signaling networks by Kaposi's sarcoma-associated herpesvirus. Biol Chem 2017

6. Dittmer DP, Damania B: Kaposi sarcoma-associated herpesvirus: immunobiology, oncogenesis, and therapy. J Clin Invest 2016, 126:3165-3175.

7. Abere B, Schulz TF: KSHV non-structural membrane proteins involved in the activation of intracellular signaling pathways and the pathogenesis of Kaposi's sarcoma. Curr Opin Virol 2016, 20:11-19.

8. Gramolelli S, Schulz TF: The role of Kaposi sarcoma-associated herpesvirus in the pathogenesis of Kaposi sarcoma. $J$ Pathol 2015, 235:368-380.

9. Schulz TF, Cesarman E: Kaposi Sarcoma-associated Herpesvirus: mechanisms of oncogenesis. Curr Opin Virol 2015, 14:116-128

10. Mendez JC, Paya CV: Kaposi's sarcoma and transplantation. Herpes 2000, 7:18-23.

11. Wood NH, Feller L: The malignant potential of HIV-associated Kaposi sarcoma. Cancer Cell Int 2008, 8:14.

12. Gessain A, Duprez R: Spindle cells and their role in Kaposi's sarcoma. Int J Biochem Cell Biol 2005, 37:2457-2465.

13. Wang HW, Trotter MW, Lagos D, Bourboulia D, Henderson S, Makinen T, Elliman S, Flanagan AM, Alitalo K, Boshoff C: Kaposi sarcoma herpesvirus-induced cellular reprogramming contributes to the lymphatic endothelial gene expression in Kaposi sarcoma. Nat Genet 2004, 36:687-693.

14. Cheng F, Pekkonen P, Laurinavicius S, Sugiyama N, Henderson S

- Gunther T, Rantanen V, Kaivanto E, Aavikko M, Sarek G et al: KSHV-initiated notch activation leads to membrane-type-1 matrix metalloproteinase-dependent lymphatic endothelialto-mesenchymal transition. Cell Host Microbe 2011, 10: 577-590.

This study describes the trans-differentiation program activated by KSHV and the tumorigenic properties of KSHV-infected LECs.

15. Weich HA, Salahuddin SZ, Gill P, Nakamura S, Gallo RC, Folkmann J: AIDS-associated Kaposi's sarcoma-derived cells in long-term culture express and synthesize smooth muscle alpha-actin. Am J Pathol 1991, 139:1251-1258.

16. Pyakurel P, Pak F, Mwakigonja AR, Kaaya E, Heiden T, Biberfeld P Lymphatic and vascular origin of Kaposi's sarcoma spindle cells during tumor development. Int J Cancer 2006, 119 : 1262-1267.

17. Simonart $T$, Degraef $C$, Heenen $M$, Hermans $P$, Van Vooren JP, Noel JC: Expression of the fibroblast/macrophage marker 1B10 by spindle cells in Kaposi's sarcoma lesions and by Kaposi's sarcoma-derived tumor cells. J Cutan Pathol 2002, 29:72-78.

18. Sturzl M, Brandstetter H, Zietz C, Eisenburg B, Raivich G, Gearing DP, Brockmeyer NH, Hofschneider PH: Identification of interleukin-1 and platelet-derived growth factor-B as major mitogens for the spindle cells of Kaposi's sarcoma: a combined in vitro and in vivo analysis. Oncogene 1995, 10:2007-2016.

19. Cancian L, Hansen A, Boshoff C: Cellular origin of Kaposi's sarcoma and Kaposi's sarcoma-associated herpesvirusinduced cell reprogramming. Trends Cell Biol 2013, 23:421-432.

20. Ojala PM, Schulz TF: Manipulation of endothelial cells by KSHV:

-• implications for angiogenesis and aberrant vascular differentiation. Semin Cancer Biol 2014, 26:69-77.

This review highlights the most relevant aspect of KSHV-induced transdifferentiation of endothelial cells.

21. Wang L, Damania B: Kaposi's sarcoma-associated herpesvirus confers a survival advantage to endothelial cells. Cancer Res 2008, 68:4640-4648.

22. Gasperini P Espigol-Frigole G, McCormick PJ, Salvucci O, Maric D, Uldrick TS, Polizzotto MN, Yarchoan R, Tosato G: Kaposi sarcoma herpesvirus promotes endothelial-to-mesenchymal 
transition through Notch-dependent signaling. Cancer Res 2012, 72:1157-1169.

23. Cheng F, Pekkonen P, Ojala PM: Instigation of Notch signaling in the pathogenesis of Kaposi's sarcoma-associated herpesvirus and other human tumor viruses. Future Microbiol 2012, 7:1191-1205

24. Radu O, Pantanowitz L: Kaposi sarcoma. Arch Pathol Lab Med 2013, 137:289-294.

25. Pantanowitz L, Dezube BJ: Kaposi sarcoma in unusual locations. BMC Cancer 2008, 8:190.

26. Dorfman RF: Kaposi's sarcoma: evidence supporting its origin from the lymphatic system. Lymphology 1988, 21:45-52.

27. Dupin N, Fisher C, Kellam P, Ariad S, Tulliez M, Franck N, van Marck E, Salmon D, Gorin I, Escande JP et al.: Distribution of human herpesvirus-8 latently infected cells in Kaposi's sarcoma, multicentric Castleman's disease, and primary effusion lymphoma. Proc Natl Acad Sci U S A 1999, 96:4546-4551.

28. Benevenuto de Andrade BA, Ramirez-Amador V, Anaya-

- Saavedra G, Martinez-Mata G, Fonseca FP, Graner E, Paes de Almeida O: Expression of PROX-1 in oral Kaposi's sarcoma spindle cells. J Oral Pathol Med 2014, 43:132-136.

In this study, the expression of prox 1 was investigated in oral KS tissues.

29. Della Bella S, Taddeo A, Calabro ML, Brambilla L, Bellinvia M,

-• Bergamo E, Clerici M, Villa ML: Peripheral blood endothelia progenitors as potential reservoirs of Kaposi's sarcomaassociated herpesvirus. PLOS ONE 2008, 3:e1520.

This study describes for the first time the properties of KSHV infected ECPs.

30. Calcaterra F, Brambilla L, Colombo E, Tourlaki A, Veraldi S,

- Carenza C, Mavilio D, Della Bella S: Increased frequency and vasculogenic potential of endothelial colony-forming cells in patients with Kaposi's sarcoma. J Invest Dermatol 2017.

This study describes the enhanced tumorigenic properties of KSHVinfected ECPs isolated from KS patients.

31. Browning PJ, Sechler JM, Kaplan M, Washington RH, Gendelman R, Yarchoan R, Ensoli B, Gallo RC: Identification and culture of Kaposi's sarcoma-like spindle cells from the peripheral blood of human immunodeficiency virus-1-infected individuals and normal controls. Blood 1994, 84:2711-2720.

32. Douglas JL, Gustin JK, Dezube B, Pantanowitz JL, Moses AV: Kaposi's sarcoma: a model of both malignancy and chronic inflammation. Panminerva Med 2007, 49:119-138.

33. Hong YK, Harvey N, Noh YH, Schacht V, Hirakawa S, Detmar M, Oliver G: Prox1 is a master control gene in the program specifying lymphatic endothelial cell fate. Dev Dyn 2002, 225:351-357.

34. Wigle JT, Harvey N, Detmar M, Lagutina I, Grosveld G, Gunn MD, Jackson DG, Oliver G: An essential role for Prox1 in the induction of the lymphatic endothelial cell phenotype. EMBO J 2002, 21:1505-1513.

35. Wigle JT, Oliver G: Prox1 function is required for the development of the murine lymphatic system. Cell 1999, 98:769-778.

36. Bixel MG, Adams RH: Master and commander: continued expression of Prox1 prevents the dedifferentiation of lymphatic endothelial cells. Genes Dev 2008, 22:3232-3235.

37. Johnson NC, Dillard ME, Baluk P, McDonald DM, Harvey NL, Frase SL, Oliver G: Lymphatic endothelial cell identity is reversible and its maintenance requires Prox1 activity. Genes Dev 2008, 22:3282-3291.

38. Petrova TV, Makinen T, Makela TP, Saarela J, Virtanen I, Ferrell RE, Finegold DN, Kerjaschki D, Yla-Herttuala S, Alitalo K: Lymphatic endothelial reprogramming of vascular endothelial cells by the Prox-1 homeobox transcription factor. EMBO J 2002, 21 4593-4599.

39. Hong YK, Foreman K, Shin JW, Hirakawa S, Curry CL, Sage DR Libermann T, Dezube BJ, Fingeroth JD, Detmar M: Lymphatic reprogramming of blood vascular endothelium by Kaposi sarcoma-associated herpesvirus. Nat Genet 2004, 36:683-685.
40. Carroll PA, Brazeau E, Lagunoff M: Kaposi's sarcomaassociated herpesvirus infection of blood endothelial cells induces lymphatic differentiation. Virology 2004, 328:7-18.

41. Emuss V, Lagos D, Pizzey A, Gratrix F, Henderson SR, Boshoff C: KSHV manipulates Notch signaling by DLL4 and JAG1 to alter cell cycle genes in lymphatic endothelia. PLoS Pathog 2009, 5: e1000616.

42. Hansen A, Henderson S, Lagos D, Nikitenko L, Coulter E, Roberts S, Gratrix F, Plaisance K, Renne R, Bower M et al.: KSHVencoded miRNAs target MAF to induce endothelial cell reprogramming. Genes Dev 2010, 24:195-205.

43. Mocroft A, Youle M, Gazzard B, Morcinek J, Halai R, Phillips AN Anti-herpesvirus treatment and risk of Kaposi's sarcoma in HIV infection. Royal Free/Chelsea and Westminster Hospitals Collaborative Group. AIDS 1996, 10:1101-1105.

44. Spano JP, Salhi Y, Costagliola D, Rozenbaum W, Girard PM: Factors predictive of disease progression and death in AIDSrelated Kaposi's sarcoma. HIV Med 2000, 1:232-237.

45. Glesby MJ, Hoover DR, Weng S, Graham NM, Phair JP, Detels R, Ho M, Saah AJ: Use of antiherpes drugs and the risk of Kaposi's sarcoma: data from the Multicenter AIDS Cohort Study. J Infect Dis 1996, 173:1477-1480.

46. Staskus KA, Zhong W, Gebhard K, Herndier B, Wang H, Renne R, Beneke J, Pudney J, Anderson DJ, Ganem D et al.: Kaposi's sarcoma-associated herpesvirus gene expression in endothelial (spindle) tumor cells. J Virol 1997, 71:715-719.

47. Cavallin LE, Goldschmidt-Clermont P, Mesri EA: Molecular and cellular mechanisms of KSHV oncogenesis of Kaposi's sarcoma associated with HIV/AIDS. PLoS Pathog 2014, 10: e1004154.

48. Mesri EA, Cavallin LE, Ashlock BM, Leung HJ, Ma Q, GoldschmidtClermont PJ: Molecular studies and therapeutic targeting of Kaposi's sarcoma herpesvirus (KSHV/HHV-8) oncogenesis. Immunol Res 2013, 57:159-165.

49. Glaunsinger B, Ganem D: Lytic KSHV infection inhibits host

-. gene expression by accelerating global mRNA turnover. MO Cell 2004, 13:713-723.

In this study, the ability of KSHV to inhibit host gene expression during the lytic reactivation is described.

50. Glaunsinger B, Ganem D: Highly selective escape from KSHV-

- mediated host mRNA shutoff and its implications for viral pathogenesis. J Exp Med 2004, 200:391-398.

This study identifies rare subsets to host transcripts that escape the host shut-off induced during KSHV lytic cycle.

51. Chandriani S, Ganem D: Host transcript accumulation during lytic KSHV infection reveals several classes of host responses PLOS ONE 2007, 2:e811.

52. Grundhoff A, Ganem D: Inefficient establishment of KSHV

-. latency suggests an additional role for continued lytic replication in Kaposi sarcoma pathogenesis. J Clin Invest 2004, 113:124-136.

The loss of KSHV episome during replication of latently infected cells in several cell types suggests a new role of lytic replication in maintaining a population of infected cells.

53. Lagunoff M, Bechtel J, Venetsanakos E, Roy AM, Abbey N, Herndier B, McMahon M, Ganem D: De novo infection and serial transmission of Kaposi's sarcoma-associated herpesvirus in cultured endothelial cells. J Virol 2002, 76:2440-2448.

54. McAllister SC, Moses AV: Endothelial cell- and lymphocytebased in vitro systems for understanding KSHV biology. Curr Top Microbiol Immunol 2007, 312:211-244.

55. Sturzl M, Gaus D, Dirks WG, Ganem D, Jochmann R: Kaposi's sarcoma-derived cell line SLK is not of endothelial origin, but is a contaminant from a known renal carcinoma cell line. Int $J$ Cancer 2013, 132:1954-1958.

56. Renne R, Blackbourn D, Whitby D, Levy J, Ganem D: Limited transmission of Kaposi's sarcoma-associated herpesvirus in cultured cells. J Virol 1998, 72:5182-5188.

57. Drexler HG, Uphoff CC, Gaidano G, Carbone A: Lymphoma cell lines: in vitro models for the study of HHV-8+ primary effusion 
lymphomas (body cavity-based lymphomas). Leukemia 1998, 12:1507-1517.

58. Mutlu AD, Cavallin LE, Vincent L, Chiozzini C, Eroles P, Duran EM, Asgari Z, Hooper AT, La Perle KM, Hilsher C et al:: In vivorestricted and reversible malignancy induced by human herpesvirus-8 KSHV: a cell and animal model of virally induced Kaposi's sarcoma. Cancer Cell 2007, 11:245-258.

59. Austgen K, Oakes SA, Ganem D: Multiple defects, including premature apoptosis, prevent Kaposi's sarcoma-associated herpesvirus replication in murine cells. J Virol 2012, 86: 1877-1882.

60. Chang HH, Ganem D: A unique herpesviral transcriptional

-• program in KSHV-infected lymphatic endothelial cells leads to mTORC1 activation and rapamycin sensitivity. Cell Host Microbe 2013, 13:429-440.

In this study, a new KSHV-induced transcriptional program that includes the expression of both latent and lytic genes without inducing cell death is described in KSHV-infected LECs.

61. Yogev O, Boshoff C: Redefining KSHV latency. Cell Host Microbe 2013, 13:373-374.

62. Hanahan D, Weinberg RA: Hallmarks of cancer: the next generation. Cell 2011, 144:646-674.

63. Weitzman MD, Carson CT, Schwartz RA, Lilley CE: Interactions of viruses with the cellular DNA repair machinery. DNA Repair (Amst) 2004, 3:1165-1173.

64. Hollingworth R, Grand RJ: Modulation of DNA damage and repair pathways by human tumour viruses. Viruses 2015 , 7:2542-2591.
65. Koopal S, Furuhjelm JH, Jarviluoma A, Jaamaa S, Pyakurel P,

-• Pussinen C, Wirzenius M, Biberfeld P, Alitalo K, Laiho M et al.: Viral oncogene-induced DNA damage response is activated in Kaposi sarcoma tumorigenesis. PLoS Pathog 2007, 3 : 1348-1360.

In this study for the first time an activated DDR was shown in KS lesions.

66. Singh VV, Dutta D, Ansari MA, Dutta S, Chandran B: Kaposi's sarcoma-associated herpesvirus induces the ATM and H2AX DNA damage response early during de novo infection of primary endothelial cells, which play roles in latency establishment. J Virol 2014, 88:2821-2834.

67. Jackson BR, Noerenberg M, Whitehouse A: A novel mechanism inducing genome instability in Kaposi's sarcoma-associated herpesvirus infected cells. PLOS Pathog 2014, 10:e1004098.

68. Hollingworth R, Skalka GL, Stewart GS, Hislop AD, Blackbourn DJ, Grand RJ: Activation of DNA damage response pathways during lytic replication of KSHV. Viruses 2015, 7:2908-2927.

69. Balistreri G, Viiliainen J, Turunen M, Diaz R, Lyly L, Pekkonen P, Rantala J, Ojala K, Sarek G, Teesalu M et al.: Oncogenic herpesvirus utilizes stress-induced cell cycle checkpoints for efficient lytic replication. PLOS Pathog 2016 12:e1005424.

70. Hosseinipour MC, Sweet KM, Xiong J, Namarika D, Mwafongo A

.• Nyirenda M, Chiwoko L, Kamwendo D, Hoffman I, Lee J et al.: Viral profiling identifies multiple subtypes of Kaposi's sarcoma. MBio 2014, 5 e01633-01614.

In this study, the transcriptional analysis of KS lesions from different patients revealed that different viral expression profiles are present. 